\title{
Complicação Decorrente da Presença de Brônquio Traqueal em Lactente. Relato de Caso *
}

\section{Complication Related to Tracheal Bronchus in Infant. Case Report}

Jaqueline Costa Reis ${ }^{1}$; César Romão Martins, TSA ${ }^{2}$; Luiz Hirotoshi Otta ${ }^{3}$; Maria Angela Tardelli, TSA ${ }^{4}$; José Luiz Gomes do Amaral, TSA ${ }^{5}$

\section{RESUMO}

Reis JC, Martins CR, Otta LH, Tardelli MA, Amaral JLG - Complicação Decorrente da Presença de Brônquio Traqueal em Lactente. Relato de Caso

JUSTIFICATIVA E OBJETIVOS: O brônquio traqueal é uma anomalia congênita com incidência aproximada de $2 \%$ na população geral, habitualmente assintomática e de diagnóstico usualmente incidental. Esta anomalia caracteriza-se pela presença de brônquio para o lobo superior direito emergindo diretamente da traquéia, junto à carina. Em vigência de intubação traqueal, este brônquio pode ter sua luz obliterada pela cânula, levando a atelectasia isolada do lobo superior direito. O objetivo deste artigo foi relatar um caso de atelectasia isolada de lobo superior direito em lactente, percebida após intubação orotraqueal, bem como fazer uma revisão de literatura sobre esta anomalia, destacando suas implicações anestésicas.

RELATO DO CASO: Paciente do sexo masculino, pardo, 5 meses de idade, $5 \mathrm{~kg}$, estado físico ASA I, portador de fístula anorretal, em programação para correção da fístula (Mini-Peña). Após intubação orotraqueal realizou-se punção de veia subclávia direita, que foi seguida de dessaturação e diminuição do murmúrio vesicular no ápice direito. As hipóteses iniciais foram de hemotórax, pneumotórax, secreção brônquica e intubação seletiva. Foi realizada radiografia de tórax, que mostrou atelectasia de lobo superior direito. Realizada broncoscopia, que revelou brônquio traqueal. A cânula foi reposicionada, com re-expansão do lobo atelectasiado.

CONCLUSÕES: Por ser uma anomalia de incidência relativamente alta (2\%), o brônquio traqueal deve ser incluído entre os diagnósticos diferenciais de atelectasia de lobo superior direito.

Unitermos: ANESTESIA, Pediátrica; COMPLICAÇÕES: brônquio traqueal

\footnotetext{
* Recebido da (Received from) Universidade Federal de São Paulo Escola Paulista de Medicina (UNIFESP - EPM), São Paulo, SP

1. Anestesiologista do Hospital São Paulo - UNIFESP - EPM

2. Anestesiologista, Preceptor dos Residentes do CET da UNIFESP - EPM

3. Médico Pneumologista - Chefe do Setor de Broncoscopia da UNIFESP EPM

4. Professora Adjunta da Disciplina de Anestesiologia, Dor e Terapia Intensiva da UNIFESP - EPM

5. Professor Titular da Disciplina de Anestesiologia, Dor e Terapia Intensiva da UNIFESP - EPM
}

Apresentado (Submitted) em13 de abril de 2005

Aceito (Accepted) para publicação em 14 de dezembro de 2005

Endereço para correspondência (Correspondence to)

Dra. Maria Angela Tardelli

Rua Napoleão de Barros, 715 - $4^{\circ}$ Andar

04024-002 São Paulo, SP

E-mail: tardelli.dcir@epm.br

(C) Sociedade Brasileira de Anestesiologia, 2006

\section{SUMMARY}

Reis JC, Martins CR, Otta LH, Tardelli MA, Amaral JLG - Complication Related to Tracheal Bronchus in Infant. Case Report

BACKGROUND AND OBJECTIVES: Tracheal bronchus is a congenital abnormality affecting approximately $2 \%$ of general population, usually asymptomatic and of incidental diagnosis. It is characterized by the presence of the bronchus to the right upper lobe emerging directly from the trachea, close to the carina. Tracheal tube may obliterate its lumen, leading to right upper lobe atelectasis. This article aimed at reporting a case of right upper lobe atelectasis in infant, noticed after tracheal intubation, and also presenting a literature review on this abnormality, highlighting its anesthetic implications.

CASE REPORT: Male patient, mulatto, 5 months old, $5 \mathrm{~kg}$, physical status ASA I, scheduled for anorectal fistula correction (Mini-Peña). Right subclavian vein was punctured after tracheal intubation, followed by desaturation and decreased right apex vesical murmur. Initial hypotheses were hemothorax, pneumothorax, bronchial secretion and selective intubation. Chest $X$-rays have shown right upper lobe atelectasis. Bronchoscopy revealed tracheal bronchus. Tube was repositioned with re-expansion of the affected lobe.

CONCLUSIONS: Because of its relatively high incidence (2\%), tracheal bronchus should be included among right upper lobe atelectasis differential diagnoses.

Key Words: ANESTHESIA, Pediatric; COMPLICATIONS: tracheal bronchus

\section{INTRODUÇÃO}

A s anomalias da árvore traqueobrônquica cursam habituAalmente assintomáticas em pacientes não anestesiados. Estas anomalias costumam ser evidenciadas na vigência de intubação traqueal, quando ocorre obstrução da saída do brônquio anômalo pela cânula. Este relato teve por objetivo descrever um caso de uma criança assintomática, do ponto de vista respiratório, que desenvolveu atelectasia isolada de lobo superior direito, após intubação orotraqueal, devido à presença de uma anomalia da árvore traqueobrônquica conhecida com brônquio traqueal, bem como fazer uma revisão sobre esta entidade clínica e suas implicações para o anestesiologista.

\section{RELATO DO CASO}

Paciente do sexo masculino, pardo, 5 meses, $5 \mathrm{~kg}$, nascido a termo (38 semanas), desenvolvimento neuropsicomotor normal, estado físico ASAI, portador de fístula anorretal, encaminhado ao centro cirúrgico para correção da anomalia. Ao exame físico apresentava-se consciente, calmo e ausculta cardiopulmonar normal. 
Foi encaminhado da enfermaria com acesso venoso $24 \mathrm{G}$ no membro superior esquerdo. Não se administrou medicação pré-anestésica.

Monitorizado com oxímetro de pulso $\left(\mathrm{SatO}_{2}\right)$, cardioscópio (FC) e pressão arterial não-invasiva (PANI). Os parâmetros iniciais foram $\mathrm{PA}=110 \times 80 \mathrm{mmHg}, \mathrm{SatO}_{2}=98 \%$ e $\mathrm{FC}=120$ bpm. Iniciada indução anestésica com oxigênio a $100 \%$ por 5 minutos. Indução venosa com alfentanil $(0,2 \mathrm{mg})$, propofol $(20 \mathrm{mg})$ e vecurônio $(0,5 \mathrm{mg})$. Realizada intubação orotraqueal com cânula 4,0 sem balonete, que apresentou vazamento durante a ventilação em sistema de Res-Baraka (Mapleson D). Optou-se pela troca por cânula 4,5 sem balonete, a intubação foi confirmada por capnografia $\left(\mathrm{P}_{\mathrm{ET}} \mathrm{CO}_{2}=30\right.$ $\mathrm{mmHg}$ ) e ausculta pulmonar bilateral. O paciente foi mantido em ventilação mecânica controlada a pressão com oxigênio: óxido nitroso 1:1 e sevoflurano. Após a indução, apresentava $\mathrm{FC}=100 \mathrm{bpm}, \mathrm{SatO}_{2}=98 \%, \mathrm{PANI}=100 \times 70 \mathrm{mmHg}$.

Pela necessidade de nutrição parenteral no pós-operatório, a equipe cirúrgica realizou punção de veia subclávia direita para inserção de cateter de duplo-lúmen. Após a passagem do cateter, houve diminuição da saturação para $93 \%$ e $\mathrm{P}_{\mathrm{ET}} \mathrm{CO}_{2}$ para $29 \mathrm{mmHg}$, com diminuição dos murmúrios vesiculares no ápice direito. Foram feitas as hipóteses de pneumotórax, secreção traqueal, intubação seletiva e/ou hemotórax. Realizada radiografia (Figura 1), que evidenciou atelectasia de lobo superior direito, o cateter de duplo-lúmen enrolado na veia subclávia direita e extremidade distal da cânula orotraqueal próxima à carina. A conduta adotada foi tração da cânula orotraqueal e do cateter central. A radiografia de controle (Figura 2) revelou persistência da imagem de atelectasia em lobo superior direito e posicionamento do cateter central e da cânula orotraqueal adequados.

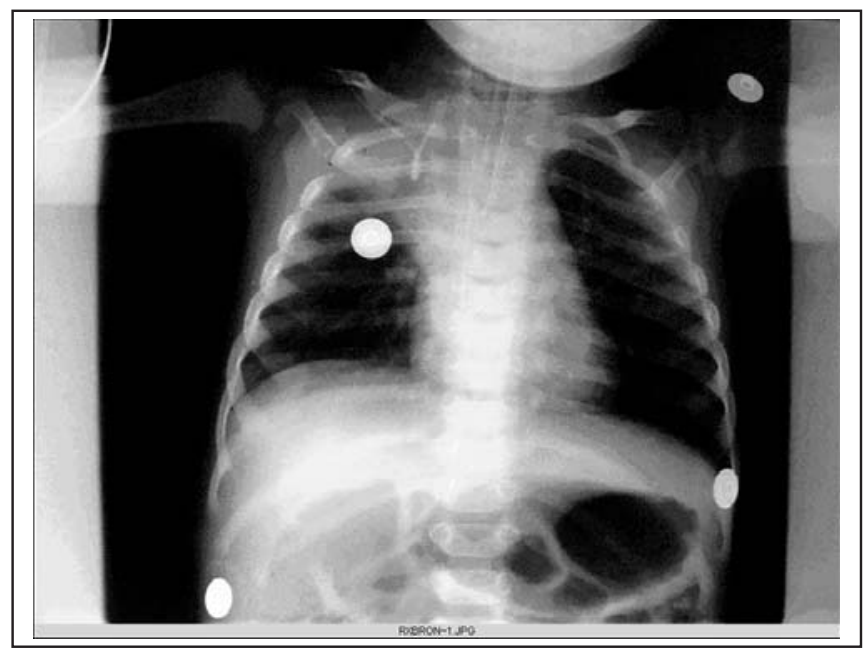

Figura 1 - Radiografia de Tórax com Atelectasia do Lobo Superior Direito

Observar o cateter de duplo-lúmen muito introduzido e enrolado na veia subclávia direita e a ponta da cânula orotraqueal junto à carina

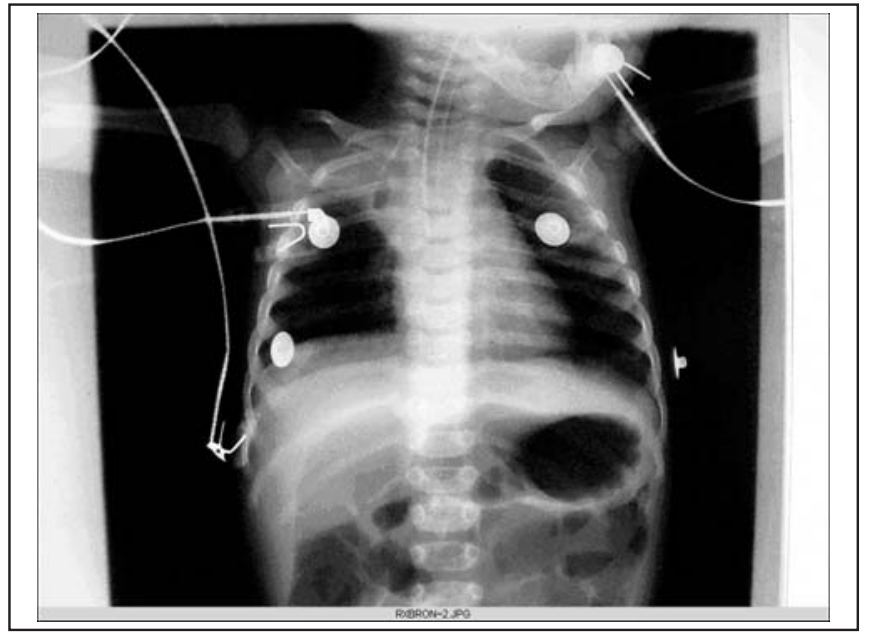

Figura 2 - Radiografia de Tórax de Controle

Observar o posicionamento adequado da cânula orotraqueal e do cateter central e persistência da atelectasia de lobo superior direito

Optou-se pela suspensão da operação e solicitou-se broncoscopia diagnóstica (Figura 3). Foi diagnosticado brônquio traqueal (anomalia congênita), em que o brônquio para o lobo superior direito emergia da parede traqueal, junto à carina, fato que resultou na sua obliteração pela cânula orotraqueal. A cânula foi reposicionada com o fibroscópio, observando-se re-expansão do lobo atelectasiado e melhora da ausculta pulmonar. Não havia secreção traqueal.

Após a broncoscopia os parâmetros foram $\mathrm{SatO}_{2}=99 \%$ e $\mathrm{P}_{\mathrm{ET}} \mathrm{CO}_{2}=36 \mathrm{mmHg}$. Não foi necessária a reversão do bloqueio neuromuscular e a extubação ocorreu sem intercorrências. O processo de diagnóstico e tratamento da atelectasia do lobo superior direito durou aproximadamente três horas e optou-se pelo adiamento da operação. O paciente foi encaminhado à sala da recuperação pós-anestésica, onde foi mantido sob nebulização com oxigênio ( 5 L. $\left.\mathrm{min}^{-1}\right)$, e parâmetros de PA $=110 \times 75 \mathrm{mmHg}, \mathrm{SatO}_{2}=97 \%$ e FC $=110 \mathrm{bpm}$. Após uma semana foi re-encaminhado ao centro cirúrgico, onde foi submetido à operação proposta anteriormente sem

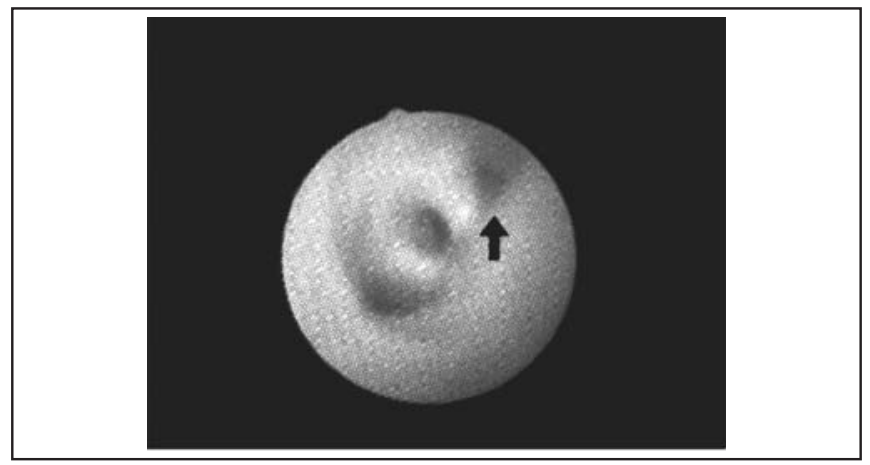

Figura 3 - Imagem da Broncoscopia, onde se visualiza Brônquio Traqueal (à direita, junto à carina principal, indicada pela seta) 
intercorrências, respeitando-se os parâmetros anatômicos da anomalia congênita.

\section{DISCUSSÃO}

O brônquio traqueal define-se como um brônquio aberrante que emerge mais freqüentemente da parede direita da traquéia, acima da carina, como resultado de um desenvolvimento anômalo no período embrionário precoce ${ }^{1}$.

Sua incidência varia de acordo com o método diagnóstico empregado. Bremer ${ }^{2}$, em 1932, em estudo anátomo-patológico, diagnosticou a anomalia em 4 de 80 fetos oriundos de abortamentos espontâneos (5\%); Inada e Kashimoto ${ }^{3}$, em 1957, relataram incidência de 0,1\% a 0,6\% em revisão da literatura japonesa; Le Roux ${ }^{4}$, em 1962, observou a anomalia presente em 30 de 1000 broncografias consecutivas (3\%); O'Sullivan e col. ${ }^{5}$, em 1998, relataram incidência de $2 \%$ em revisão de literatura, que incluía vários métodos diagnósticos, como tomografia computadorizada e broncoscopia. Acredita-se que a incidência seja em torno de $2 \%$, e que a maior incidência relatada por Bremer seja resultante do método empregado, pois é sabido que a incidência de anomalias congênitas é maior em fetos oriundos de abortamentos espontâneos.

Quanto à etiologia, Alescio e col. ${ }^{6}$ comprovaram que a formação do brônquio traqueal é produto de anormalidade na embriogênese, e não uma anomalia genética. O estudo demonstrou que o transplante de mesênquima brônquico no epitélio traqueal resultou no surgimento de brônquio traqueal.

O brônquio traqueal pode apresentar-se sob diversos padrões anatômicos. A figura 4 apresenta os padrões de brônquio traqueal à direita ${ }^{2}$. Existem também relatos de casos de brônquio para o lobo superior direito emergindo do brônquio fonte esquerdo ${ }^{7}$.

A anomalia é habitualmente assintomática, porém pode ser acompanhada de pneumonias lobares ou atelectasias de repetição no lobo superior direito ${ }^{8,9}$.

O diagnóstico geralmente é incidental e feito por broncografia, broncoscopia ou tomografia computadorizada de tórax ${ }^{8,9}$.

Habitualmente esta anomalia não está associada a outras anomalias congênitas, porém existem relatos de associação com hipoplasia traqueal ${ }^{10}$, estenose traqueal, enfisema lobar, lesões pulmonares císticas, anomalias cardiovasculares, outras anomalias da árvore traqueobrônquica e com a síndrome de Down. A presença de hipoplasia ou fusão do primeiro e segundo arcos costais, de costelas lombares bilaterais e de outras anomalias vertebrais aumenta a suspeita de brônquio traqueal ou fístula traqueo-esofágica. Anomalias

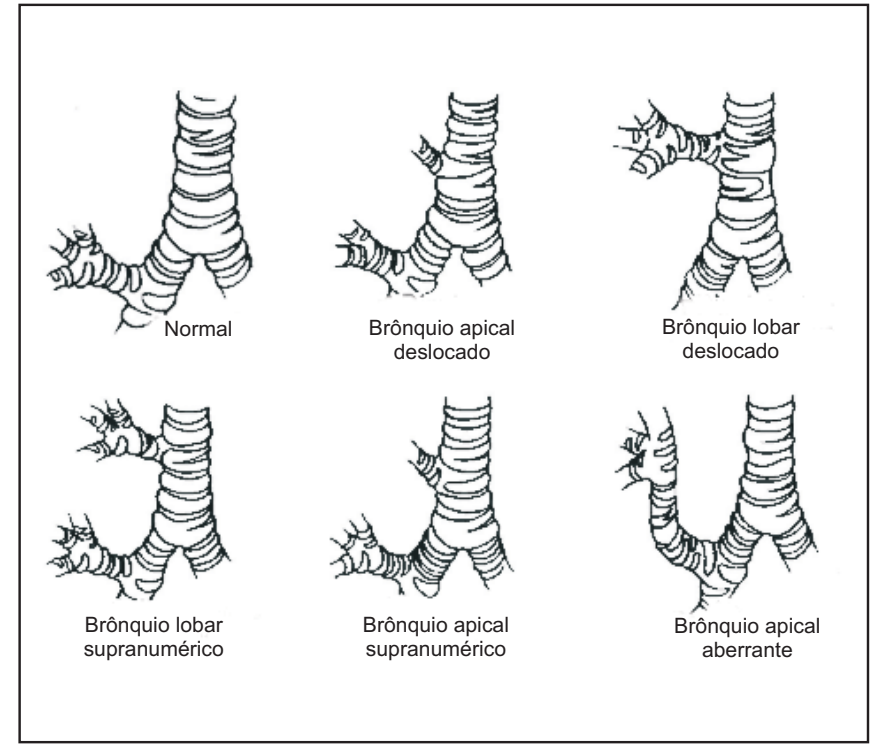

Figura 4 - Padrões de Distribuição dos Brônquios Traqueais à Direita. Adaptado de Barat e col. ${ }^{1}$

do lado esquerdo podem estar associadas a anomalias cerebrais.

O brônquio traqueal também é conhecido na literatura como brônquio de porco, uma vez que os porcos apresentam naturalmente brônquio traqueal.

Após a IOT, ocorre obstrução da saída do brônquio traqueal pela cânula atelectasiando o lobo envolvido. O diagnóstico dessa área colapsada, na criança, é difícil porque a pequena distância entre as áreas atelectasiadas e as áreas normalmente ventiladas no tórax podem confundir a ausculta, como ocorreu neste caso. No início a ausculta era aparentemente normal, provavelmente porque se auscultavam ruídos transmitidos por áreas adjacentes normais.

Os valores de pressão arterial elevada para a idade decorreram provavelmente da escolha de um manguito de tamanho inadequadamente pequeno, que superestima o real valor da pressão arterial. Por condições materiais do Centro Cirúrgico não foi possível trocar o manguito por outro mais adequado, e optou-se por acompanhar a pressão arterial por sua evolução temporal.

Este caso mostra a importância que os anestesiologistas devem dar a esta anomalia de incidência relativamente alta, habitualmente assintomática, porém que pode estar associada a outras anomalias, alertando para a possibilidade de obliteração do brônquio para o lobo superior direito após intubação traqueal, particularmente em crianças.

Por ser uma anomalia de incidência relativamente alta (2\%), o brônquio traqueal deve ser incluído entre os diagnósticos diferenciais de atelectasia de lobo superior direito. 


\section{Complication Related to Tracheal Bronchus in Infant. Case Report}

Jaqueline Costa Reis, M.D.; César Romão Martins TSA, M.D.; Luiz Hirotoshi Otta, M.D.; Maria Angela Tardelli, TSA, M.D.; José Luiz Gomes do Amaral, TSA, M.D.

\section{INTRODUCTION}

Tracheobronchial tree abnormalities are in general asymptomatic in non-anesthetized patients. These abnormalities are evidenced after tracheal intubation when the abnormal bronchus lumen can be blocked by the tube. This report aimed at describing the case of an asymptomatic child who developed right upper lobe atelectasis after tracheal intubation related to the presence of a tracheobronchial tree abnormality called tracheal bronchus, as well as presenting a literature review on this clinical entity and its anesthetic implications.

\section{CASE RE ORT}

A 5 months old male mulatto patient, $5 \mathrm{~kg}$, born at term (38 weeks), physical status ASA I and normal neuropsychomotor development, was referred to the Surgical Center for anorectal fistula correction. At physical evaluation, patient was conscious, relaxed and with normal cardiopulmonary auscultation.

The infant was brought from the ward with a $24 \mathrm{G}$ venous access on left arm and no premedication.

Monitoring consisted of pulse oximetry $\left(\mathrm{SatO}_{2}\right)$, cardioscope $(\mathrm{HR})$ and noninvasive blood pressure (NIBP). Baseline parameters were $\mathrm{BP}=110 \times 80 \mathrm{mmHg}$, SatO $\mathrm{O}_{2}=98 \%$ and $\mathrm{HR}=$ $120 \mathrm{bpm}$. Anesthesia was started with $100 \%$ oxygen for 5 minutes and intravenous alfentanil $(0.2 \mathrm{mg})$, propofol $(20 \mathrm{mg})$ and vecuronium $(0.5 \mathrm{mg})$. Tracheal intubation was performed with a 4.0 cuffless tube which allowed some leakage during ventilation in Res-Baraka system (Mapleson D). It was so replaced by a 4.5 cuffless tube, and intubation confirmed by capnography $\left(\mathrm{P}_{\mathrm{ET}} \mathrm{CO}_{2}=30 \mathrm{mmHg}\right)$ and bilateral pulmonary auscultation. Patient was maintained under pressure-controlled ventilation with 1:1 oxygen/nitrous oxide and sevoflurane. Parameters after induction were: $\mathrm{HR}=100$ bpm, SatO $_{2}=98 \%$, NIBP $=100 \times 70 \mathrm{mmHg}$.

Because of the postoperative parenteral nutrition indication, the right subclavian vein was punctured for double-lumen catheter insertion. After catheter installation, there was decreased saturation to $93 \%$ and $\mathrm{P}_{\mathrm{ET}} \mathrm{CO}_{2}$ to $20 \mathrm{mmHg}$, with decreased right apex vesical murmurs. The initial hypotheses were pneumothorax, tracheal secretion, selective intubation and/or hemothorax. -rays (Figure 1) have evidenced right upper lobe atelectasis, double lumen catheter coiled around right subclavian vein and distal tracheal tube tip close to the carina. The tracheal tube and central catheter were then both pulled backwards a little. Control -rays (Figure 2) have

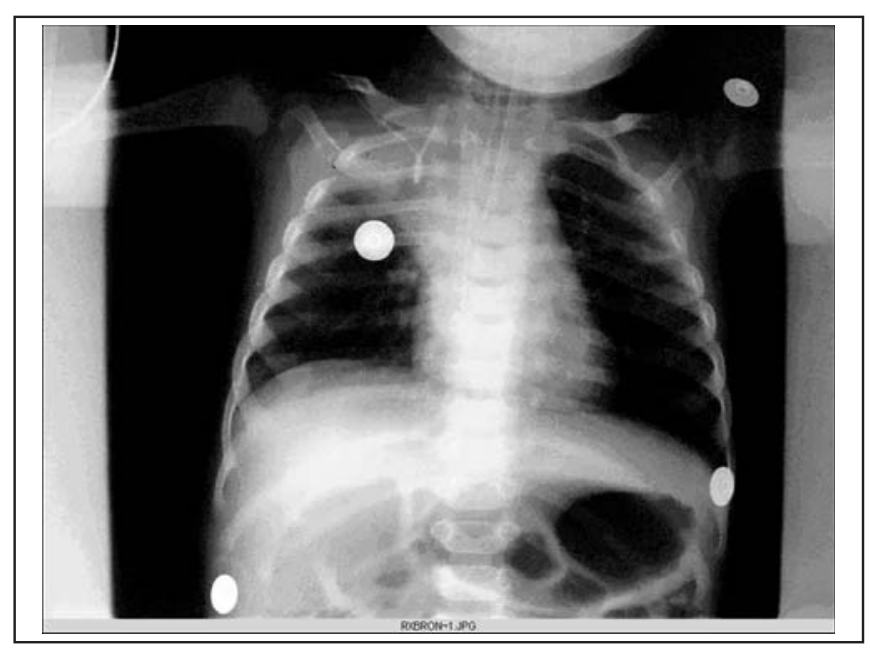

Figure 1 - Chest -Rays Showing Right Upper Lobe Atelectasis Note the double-lumen catheter too inserted and coiled around the right subclavian vein and tracheal tube tip close to carina

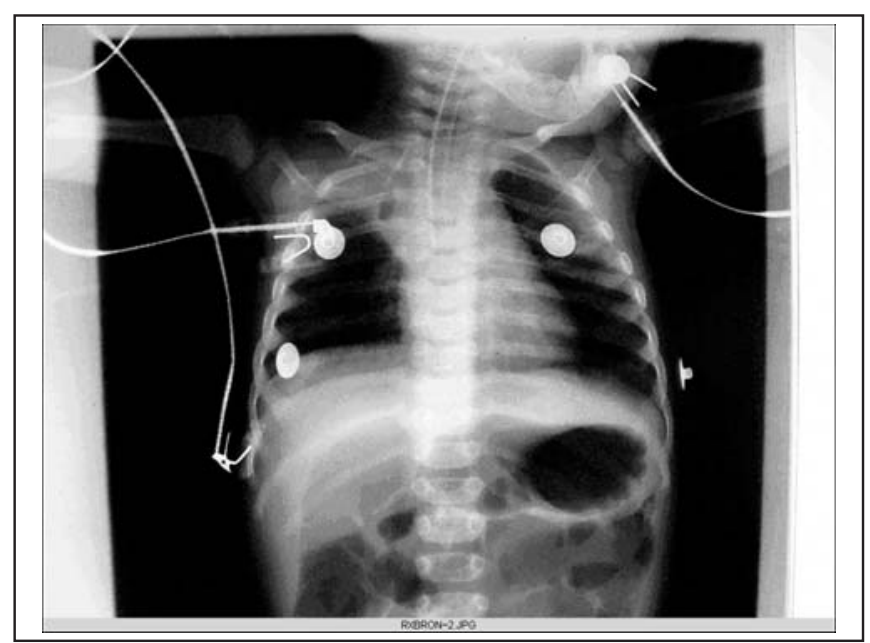

Figure 2 - Control Chest -Rays

Where there is adequate tracheal tube and central catheter positioning and persistence of right upper lobe atelectasis

shown persistence of right upper lobe atelectasis and adequate central catheter and tracheal tube positioning.

Surgery was interrupted and diagnostic bronchoscopy was requested (Figure 3). Diagnosis was tracheal bronchus (congenital abnormality), where the right upper lobe bronchus emerged from the tracheal wall, close to the carina, resulting in its obliteration by the tracheal tube. The tube was then repositioned assisted by fibroscopy, followed by re-expansion of atelectasis and improved pulmonary auscultation. There was no tracheal secretion.

Post-bronchoscopy parameters were $\mathrm{SatO}_{2}=99 \%$ and $\mathrm{P}_{\mathrm{ET}} \mathrm{CO}_{2}=36 \mathrm{mmHg}$. Neuromuscular block reversal was not needed and extubation occurred normally. Diagnosis and treatment of right upper lobe atelectasis took approximately 3 


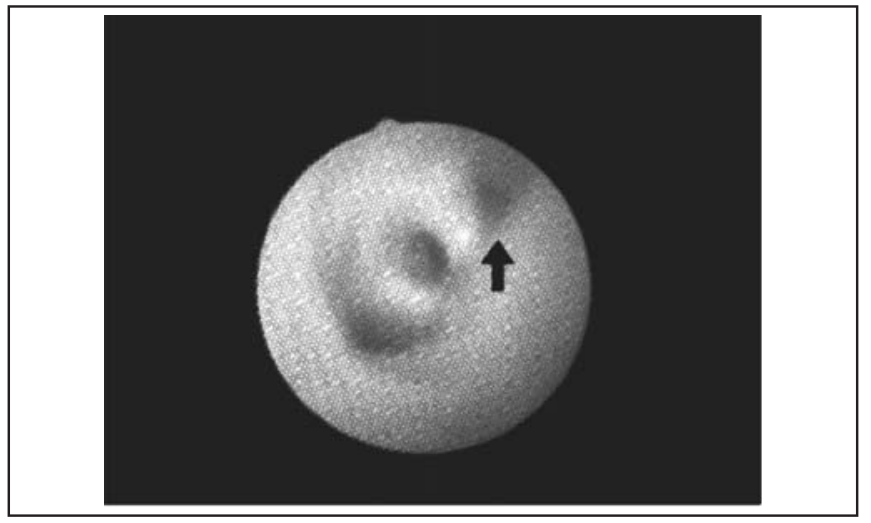

Figure 3 - Bronchoscopy Image Showing Tracheal Bronchus (to the right, close to main carina, indicated by the arrow)

hours and surgery was postponed. Patient was referred to the post-anesthetic recovery unit and was maintained under oxygen mist $\left(5 \mathrm{~L} \cdot \mathrm{min}^{-1}\right)$ with $\mathrm{SatO}_{2}=97 \%, \mathrm{BP}=110 \times 75 \mathrm{mmHg}$ and $\mathrm{HR}=110 \mathrm{bpm}$.

One week later, patient was once again referred to the Surgical Center and was submitted to the proposed procedure uneventfully, respecting the anatomic parameters of the congenital abnormality.

\section{DISCUSSION}

Tracheal bronchus is defined as an aberrant bronchus most frequently emerging from the right tracheal wall above the carina, as result of abnormal development during early embryonal period ${ }^{1}$.

Its incidence varies according to the diagnostic method. Bre$\mathrm{mer}^{2}$, in a pathological study from 1932, diagnosed the abnormality in 4 out of 80 fetuses resulting from spontaneous abortions $(5 \%)$. Inada \& Kashimoto ${ }^{3}$, in 1957, reported incidence of $0.1 \%$ to $0.6 \%$ after Japanese literature review. Le Roux ${ }^{4}$, in 1962 , observed the abnormality in 30 out of 1000 consecutive bronchographies (3\%). O'Sullivan et al. ${ }^{5}$, in 1998 , reported $2 \%$ incidence in a literature review including several diagnostic methods such as CT and bronchoscopy. It is accepted that the overall incidence is approximately $2 \%$ and that the higher number reported by Bremer is a result of his method, since it is well known that congenital abnormalities are present in higher incidence in fetuses resulting from spontaneous abortions.

Regarding the etiology, Alescio et al. ${ }^{6}$ have proven that tracheal bronchus formation is an embryogenesis defect rather than a genetic abnormality. The study has shown that bronchial mesenchyma transplantation in the tracheal epithelium has resulted in tracheal bronchus.

Tracheal bronchi may present with different anatomic patterns. Figure 4 shows right tracheal bronchus patterns ${ }^{2}$. There are also reports on right upper lobe bronchus emerging from the left main bronchus ${ }^{7}$.

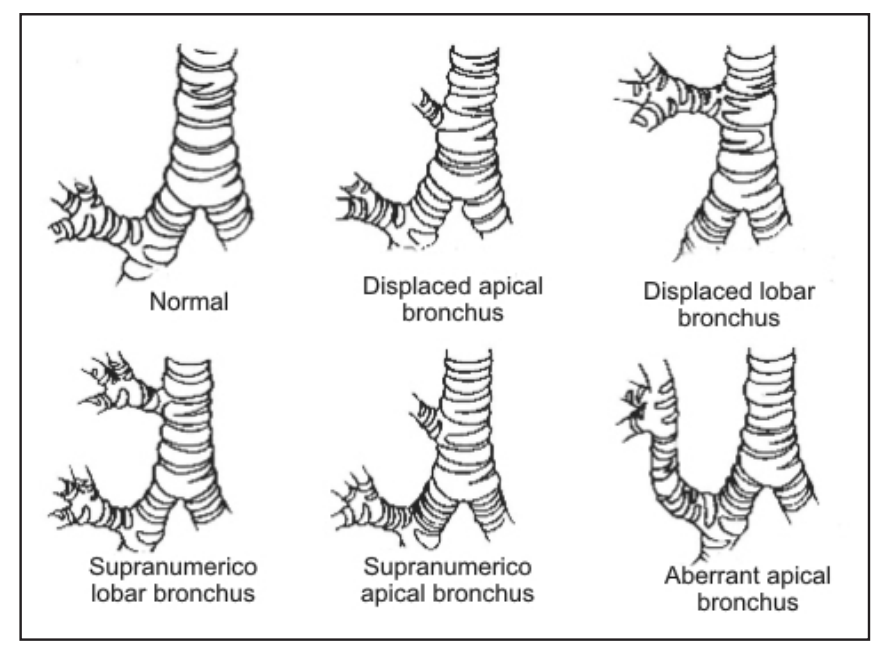

Figure 4 - Tracheal Bronchi Distribution Patterns to the Right. Adapted from Barat et al.

This condition is usually asymptomatic, but may be followed by lobe pneumonias or right upper lobe repetition atelectasis ${ }^{8,9}$.

Diagnosis is often incidental and achieved by bronchography, bronchoscopy or chest $\mathrm{CT}^{8,9}$.

It is commonly not associated to other congenital anomalies; however there are reports ${ }^{10}$ of associated tracheal hypoplasia, tracheal stenosis, lobe emphysema, cystic pulmonary lesions, cardiovascular disorders, other bronchial tree disorders and Down syndrome. The presence of hypoplasia, or fusion of first and second costal arches, bilateral lumbar ribs and other vertebral abnormalities increases the suspicion of tracheal bronchus or tracheo-esophageal fistula. Left abnormalities may be associated to brain anomalies.

Tracheal bronchus is also known as swine's bronchus since swine have natural tracheal bronchus.

The tracheal tube can obliterate the tracheal bronchus lumen, leading to atelectasis of the involved lobe. Diagnosis of this collapsed area in children is difficult because the short distance between atelectasis areas and normally ventilated areas may mislead auscultation, as in this case. In the beginning, auscultation was apparently normal, probably due to transmission of sounds from adjacent areas.

High blood pressure values for the age were probably caused by the choice of inadequately short cuff, which overestimates real blood pressure value. As we couldn't replace it at that time, we decided to follow blood pressure by its temporal evolution.

This case reinforces the attention to be given by anesthesiologists to this abnormality of relatively high incidence, warning for the possibility of bronchial obliteration to the right upper lobe after tracheal intubation, especially in pediatric patients. 
Because its relatively high incidence $(2 \%)$, tracheal bronchus should be included among right upper lob atelectasis differential diagnoses.

\section{REFERÊNCIAS - REFERENCES}

01. Barat M, Konrad HR - Tracheal bronchus. Am J Otolayngol, 1987;8:118-122.

02. Bremer JL - Accessory bronchi in embryos: their occurrence and probable fate. Anat Rec, 1932;79:228-234.

03. Inada K, Kashimoto S - An anomalous tracheal bronchus to the right upper lobe. Dis Chest, 1957;140:671-674.

04. le Roux BT - Anatomical abnormalities of the right upper bronchus. J Thorac Cardiovasc Surg, 1962;44:225-227.

05. O'Sullivan BP, Frassica JJ, Rayder SM - Tracheal bronchus: a cause of prolonged atelectasis in intubated children. Chest, 1998;113:537-540.

06. Alescio T, Cassini A- Introduction in vitro of tracheal buds by pulmonary mesenchyme grafted on tracheal epithelium. J Exp Zool, 1962;150:83-94.

07. Laforet EG, Starkey GW, Scheff S - Anomalies of upper lobe bronchial distribution. J Thorac Cardiovasc Surg, 1962;43: 595-606.

08. Ikeno S, Mitsuhata $\mathrm{H}$, Hirabayashi $\mathrm{Y}$ et al - Airway management for patients with a tracheal bronchus. Br J Anesth, 1996;76: 573-575.

09. Vredevoe LA, Brechner T, Moy P - Obstruction of anomalous tracheal bronchus with endotracheal intubation. Anesthesiology, 1981;55:581-583

10. Weigel W, Kaufmann HJ - The frequency and types of other congenital anomalies in association with tracheoesophagel malformations. Radiologic study of 83 such infants. Clin Pediatr, 1976;15:819-834

\section{RESUMEN}

Reis JC, Martins CR, Otta LH, Tardelli MA, Amaral JLG - Complicación Debida a la Presencia de BronquioTraqueal en un Lactante. Relato de Caso

JUSTIFICATIVA Y OBJETIVOS: EI bronquio traqueal es una anomalía congénita con incidencia de aproximadamente $2 \%$ en la población y cuyo diagnóstico habitual es incidental. Se caracteriza por la emergencia del bronquio para el lóbulo superior derecho directamente desde la traquea, próximo a la carina. Al proceder a la intubación traqueal su luz puede ser ocluida por la sonda y ser causa de atelectasia exclusiva del lóbulo superior derecho. El objetivo de este artículo es relatar un caso de atelectasia del lóbulo superior derecho en un lactante, solo percibida después de la intubación traqueal y hacer una revisión de la literatura sobre este tópico destacando su importancia para la anestesia.

RELATO DEL CASO: Paciente masculino, pardo, 5 meses de edad y $5 \mathrm{~kg}$ de peso, estado físico ASA I, portador de fístula ano-rectal, programado para corrección de la fístula con la técnica de mini-Peña. Luego de la intubación traqueal se realizó punción de la vena subclavia derecha, ocurriendo caída de la saturación de $\mathrm{O}_{2}$ y disminución de ventilación en el ápice derecho. Inicialmente las hipótesis fueron hemotórax, neumotórax, secreción bronquial excesiva o intubación selectiva. Fue realizada radiografía de tórax que mostró atelectasia exclusiva del lóbulo superior derecho. La broncoscopía reveló la presencia de bronquio traqueal. La sonda traqueal fue reposicionada y el lóbulo derecho se re-expandió.

CONCLUSIONES: Como se trata de una anomalía con incidencia relativamente alta ( $2 \%$ de la población), el bronquio traqueal debe ser incluido entre los diagnósticos diferenciales en atelectasia del lóbulo superior derecho. 